\title{
MODICA TYPE GRADIENT ESTIMATES FOR AN INHOMOGENEOUS VARIANT OF THE NORMALIZED $p$-LAPLACIAN EVOLUTION
}

\author{
AGNID BANERJEE AND NICOLA GAROFALO
}

Dedicated to Enzo Mitidieri, on the occasion of his 60th birthday

\begin{abstract}
In this paper, we study an inhomogeneous variant of the normalized $p$-Laplacian evolution which has been recently treated in [BG1], [Do], [MPR] and [Ju]. We show that if the initial datum satisfies the pointwise gradient estimate (1.6) a.e., then the unique solution to the Cauchy problem (1.2) satisfies the same gradient estimate a.e. for all later times, see (1.7) below. A general pointwise gradient bound for the entire bounded solutions of the elliptic counterpart of equation (1.2) was first obtained in [CGS]. Such estimate generalizes one obtained by L. Modica for the Laplacian, and it has connections to a famous conjecture of De Giorgi.
\end{abstract}

\section{INTRODUCTION}

Recently, there has been increasing attention about the equation of the so-called normalized $p$-Laplacian evolution

$$
|D u|^{2-p} \operatorname{div}\left(|D u|^{p-2} D u\right)=u_{t}, \quad 1<p<\infty,
$$

see [BG1], [Do], [MPR], [Ju], [BG2] and [JK]. The equation (1.1) is an evolution associated with the $p$-Laplacian that interpolates between the motion by mean curvature, which corresponds to the case $p=1$, and the heat equation, corresponding to $p=2$. In the interesting paper [MPR] solutions to (1.1) have been characterized by asymptotic mean value properties. These properties are connected with the analysis of tug-of-war games with noise in which the number of rounds is bounded. The value functions for these games approximate a solution to the PDE (1.1) when the parameter that controls the size of possible steps go to zero. The equation (1.1) also arises in image processing, see [Do], in which the Cauchy-Neumann problem was studied. In [BG1] we constructed viscosity solutions to (1.1) and derived properties such as comparison principles for solutions of (1.1), convergence of solutions as $p \rightarrow 1$, and the large-time behavior of solutions to a Cauchy-Dirichlet problem for (1.1). We also proved unweighted energy monotonicity and a generalized Struwe's monotonicity formula. In the paper [Ju] Juutinen studied the large-time behavior for $p>2$ of solutions of (1.1). The case $p=\infty$ of the normalized $\infty$-Laplacian evolution was studied in $[\mathrm{JK}]$. The equation (1.1) has the advantage of being 1-homogeneous but it has the serious disadvantage of having a non-divergence structure.

In the present paper for a given $T>0$ we consider the following Cauchy problem in $\mathbb{R}^{n} \times[0, T]$

$$
\left\{\begin{array}{l}
|D u|^{2-p}\left\{\operatorname{div}\left(|D u|^{p-2} D u\right)-F^{\prime}(u)\right\}=u_{t}, \\
u(\cdot, 0)=g .
\end{array}\right.
$$

We suppose that $F \in C_{l o c}^{2, \beta}(\mathbb{R})$ for some $\beta>0$ and $F \geq 0$. Throughout this paper we assume $1<p \leq 2$. We observe that, because of its non-divergence structure, when $F \not \equiv 0$ the equation (1.2) does not make sense for $p>2$ because the term $|D u|^{2-p} F^{\prime}(u)$ cannot be interpreted in the viscosity sense at the critical point of a test function $\phi$, see Definition 2.1 below. As a

First author was supported in part by the second author's NSF Grant DMS-1001317 and by a postdoctoral grant of the Institute Mittag-Leffler.

Second author was supported in part by NSF Grant DMS-1001317 and by a grant of the University of Padova, "Progetti d'Ateneo 2013". 
consequence, in the case $p>2$ it presently remains an interesting open question what is the right evolution for which results similar to those in this paper can be established.

The equation in (1.2) can be considered as the parabolic counterpart of

$$
\operatorname{div}\left(|D u|^{p-2} D u\right)=F^{\prime}(u),
$$

which is a special case of the class of equations $\operatorname{div}\left(\Phi^{\prime}\left(|D u|^{2}\right) D u\right)=F^{\prime}(u)$ treated in [CGS]. As a consequence of the results in [CGS], it follows that entire bounded (weak) solutions to (1.3) satisfy the following pointwise gradient estimate

$$
|D u|^{p} \leq \frac{p}{p-1} F(u) .
$$

We recall that in the linear case $p=2$ the estimate (1.4) was first proved by L. Modica in [Mo]. The estimate (1.4) (in fact, a generalization of it) was employed in [CGS] to provide a partial answer to a famous conjecture of De Giorgi, see [dG] (also known as the $\varepsilon$-version of the Bernstein theorem for minimal graphs) asserting that entire solutions to

$$
\Delta u=u^{3}-u,
$$

such that $|u| \leq 1$ and $\frac{\partial u}{\partial x_{n}}>0$, must be one-dimensional, i.e., must have level sets which are hyperplanes, at least in dimension $n \leq 8$. In [CGS] the estimate (1.4) was also used to establish a result on the propagation of the zeros of a solution to (1.3). We recall that the conjecture of De Giorgi has been fully solved for $n=2$ in [GG1] and $n=3$ in [AC], and it is known to fail for $n \geq 9$, see [dPKW]. For $4 \leq n \leq 8$ it is still an open question. Additional fundamental progress on De Giorgi's conjecture is contained in the papers [GG2], [Sa].

We mention that Modica type gradient estimates have been extended to more general integrands of the calculus of variations in [DG], to domains $\Omega$ which are epigraphs with boundary having nonnegative mean curvature in $[\mathrm{FV}]$, and also to compact Riemannian manifolds which nonnegative Ricci curvature, see [FV1] and the references therein. For results concerning the $p$-Laplacian version of De Giorgi's conjecture, we refer the reader to the interesting paper [SSV]. For further results, the state of art and recent progress on De-Giorgi's conjecture, we refer to $[\mathrm{CNV}],[\mathrm{FV} 2]$ and $[\mathrm{FSV}]$ and one can find the references therein.

In this paper, we study the parabolic analogue of the Modica type gradient estimate (1.4). Before stating our main results, we introduce the relevant class of solutions for the Cauchy problem (1.2):

$$
H^{T}=\left\{u \in C\left(\mathbb{R}^{n} \times[0, T]\right) \mid x \rightarrow u(x, t) \in C^{0,1}\left(\mathbb{R}^{n}\right),\|u\|_{L^{\infty}\left(\mathbb{R}^{n} \times[0, T]\right)},\|D u\|_{L^{\infty}\left(\mathbb{R}^{n} \times[0, T]\right)}<\infty\right\} .
$$

The notation $C^{0,1}(\Omega)$ indicates the class of Lipschitz continuous functions on a given open set $\Omega \subset \mathbb{R}^{n}$. The following is our main result.

Theorem 1.1. Let $g \in C^{0,1}\left(\mathbb{R}^{n}\right)$ with $\|g\|_{L^{\infty}\left(\mathbb{R}^{n}\right)},\|D g\|_{L^{\infty}\left(\mathbb{R}^{n}\right)}<\infty$. Moreover, corresponding to $g$, we assume that $F$ satisfies the assumption (4.4) below. Then, for every $T>0$ there exists a unique solution $u$ to the Cauchy problem (1.2) in the class $H^{T}$. Furthermore, if the initial datum $g$ satisfies the following gradient estimate for a.e. $x \in \mathbb{R}^{n}$

$$
|D g(x)|^{p} \leq \frac{p}{p-1} F(g(x)),
$$

then, at any given time $t>0$ one has for a.e. $x \in \mathbb{R}^{n}$

$$
|D u(x, t)|^{p} \leq \frac{p}{p-1} F(u(x, t)) .
$$

Remark 1.2. The assumption (4.4) below is used to assert the existence of solutions in the class $H^{T}$ via a regularization scheme described in the subsequent sections, see Remark 4.1. The hypothesis (4.4) is however not needed when $1<p<2$, see Remark 4.2 below. In addition, such a regularization scheme is also crucially employed to justify the computations in Section 
5. Now, when $p=2$, any solution in the class $H^{T}$ is a classical solution, a fact which follows from the parabolic regularity theory. Hence, in this case one does not need to apply any further regularization scheme. In conclusion, if we a priori assume that the solution u belongs to the class $H^{T}$, then we obtain the following version of Theorem 1.1.

Theorem 1.3. Let $1<p \leq 2$, and for some $0<T \leq \infty$ let $u \in H^{T}$ be a solution to

$$
|D u|^{2-p}\left\{\operatorname{div}\left(|D u|^{p-2} D u\right)-F^{\prime}(u)\right\}=u_{t},
$$

where $F \in C_{\text {loc }}^{2, \beta}(\mathbb{R})$ for some $\beta>0$, and $F \geq 0$. If at some time level $t_{0} u\left(\cdot, t_{0}\right)$ satisfies $(1.7)$, then $u(\cdot, t)$ satisfies $(1.7)$ for all $t_{0} \leq t \leq T$ ( $t<\infty$ if $\left.T=\infty\right)$.

Remark 1.4. Note that unlike the hypothesis in Theorem 1.1, in Theorem 1.3 we do not require that F satisfy (4.4). See Remark 1.2 above.

Theorem 1.1 and Theorem 1.3 can be considered as a parabolic analogue in the case $1<p \leq 2$ of the above mentioned result in [CGS] ( see also [Mo]) which states that an entire bounded solution to (1.3) satisfies the estimate (1.4) except that in our situation we are only able to assert that the estimate (1.7) holds a.e. in $\mathbb{R}^{n}$. It remains an open question as to whether the solution $u$ in Theorem 1.1 has higher regularity so that one can assert that the estimate (1.7) holds pointwise everywhere. In the next result we show that, under an additional assumption on the initial datum $g$, this is true when $n=2$.

Theorem 1.5. Let $n=2$, and let $u, g$ be as in Theorem 1.1. Furthermore, if the initial datum $g$ has bounded derivatives up to order two there exists $\alpha \in(0,1)$ depending only on $p$ such the solution $u(\cdot, t) \in C^{1, \alpha}$ for every $t>0$. Consequently, the gradient estimate (1.7) holds pointwise everywhere.

We conclude with an application of the estimate (1.7). The following result can be thought of as theorem on the propagation of zeros for solutions of the Cauchy problem (1.2).

Theorem 1.6. Suppose that the initial datum g satisfies (1.6), and let $u$ be the solution as in Theorem 1.1. If $F\left(u\left(x_{0}, t_{0}\right)\right)=0$ for some point $\left(x_{0}, t_{0}\right)$, then $u\left(\cdot, t_{0}\right)$ is constant.

In closing we mention that concerning Theorems 1.1 and 1.6 and for the standard heat equation $(p=2)$, there is an unpublished note of Caffarelli, Garofalo, Segala, [CGSpc]. There, the authors could successfully employ subsolution estimates and compactness arguments based on $C^{1, \alpha}$ estimates. In our situation, however, in the genuinely nonlinear case when $p \neq 2$, the subsolutions arguments work, but only in a restricted sense, i.e., we do all our calculations at the point of extrema and substitute the undesirable higher order term in terms of lower-order terms which can be controlled. The higher- order terms show up in our situation because of the normalization of the equation which is obviously evident when $p \neq 2$. Secondly, we do not have $C^{1, \alpha}$ estimates, and we compensate for this with the use of barriers.

Acknowledgment: The paper was finalized during the first author's stay at the Institut MittagLeffler during the semester long program Homogenization and Random Phenomenon. The first author would like to thank the Institute and the organizers of the program for the kind hospitality and the excellent working conditions.

\section{Preliminaries}

Suppose that $u$ is a solution to the equation (1.2). We begin by observing that, after some formal computations, we have the following equation in non-divergence form

$$
\left(\delta_{i j}+(p-2) \frac{u_{i} u_{j}}{|D u|^{2}}\right) u_{i j}=|D u|^{2-p} f(u)+u_{t},
$$


where $f=F^{\prime}$ (see [BG1] for similar formal computations in the homogeneous case $F \equiv 0$ ). Following [CGG], we now introduce the following notion of viscosity solution to the equation in (1.2).

Definition 2.1. A function $u \in C\left(\mathbb{R}^{n} \times[0, T)\right) \cap L^{\infty}\left(\mathbb{R}^{n} \times[0, T)\right)$ is called a viscosity subsolution of $(2.1)$, provided that for every $\phi \in C^{2}(\Omega \times(0, T))$ such that

$$
u-\phi \quad \text { has a local maximum at } z_{0} \in \Omega \times(0, T),
$$

then either

$$
\left\{\begin{array}{l}
\phi_{t}+|D \phi|^{2-p} f(u) \leq\left(\delta_{i j}+(p-2) \frac{\phi_{i} \phi_{j}}{|D \phi|^{2}}\right) \phi_{i j} \quad \text { at } z_{0}, \\
\text { if } D \phi\left(z_{0}\right) \neq 0,
\end{array}\right.
$$

or

$$
\left\{\begin{array}{l}
\inf _{|a|=1}\left\{\phi_{t}+|D \phi|^{2-p} f(u)-\left(\delta_{i j}+(p-2) a_{i} a_{j}\right) \phi_{i j}\right\} \leq 0 \quad \text { at } z_{0}, \\
\text { if } D \phi\left(z_{0}\right)=0 .
\end{array}\right.
$$

A function $u$ is a viscosity supersolution if $v=-u$ is a viscosity subsolution. Finally, $u$ is a viscosity solution if it is at the same time a subsolution and a supersolution.

Similarly to the case $F=0$, by arguing as in Proposition 2.8 in [BG1] we have the following equivalent definition.

Definition 2.2. A function $u \in C\left(\mathbb{R}^{n} \times[0, T)\right) \cap L^{\infty}\left(\mathbb{R}^{n} \times[0, T)\right)$ is called a viscosity subsolution of (2.1), provided that for every $\phi \in C^{2}(\Omega \times(0, T))$ such that

$$
u-\phi \quad \text { has a local maximum at } z_{0} \in \Omega \times(0, T) \text {, }
$$

then

$$
\left\{\begin{array}{l}
\phi_{t}+|D \phi|^{2-p} f(u) \leq\left(\delta_{i j}+(p-2) \frac{\phi_{i} \phi_{j}}{|D \phi|^{2}}\right) \phi_{i j} \quad \text { at } \quad z_{0}, \\
\text { if } D \phi\left(z_{0}\right) \neq 0
\end{array}\right.
$$

or

$$
\left\{\begin{array}{l}
\phi_{t}+|D \phi|^{2-p} f(u) \leq\left(\delta_{i j}+(p-2) a_{i} a_{j}\right) \phi_{i j} \quad \text { at } z_{0}, \\
\text { for some } \quad a \in \mathbb{R}^{n} \quad \text { with } \quad|a| \leq 1, \text { if } D \phi\left(z_{0}\right)=0 .
\end{array}\right.
$$

Analogous definitions for supersolutions, and for solution.

\section{Maximum modulus PRINCIPLe}

In this short section we establish a maximum modulus theorem for viscosity solutions of (1.2) which will be needed subsequently.

Theorem 3.1. Let $u$ and $v$ be two bounded continuous solutions in $\mathbb{R}^{n} \times[0, T]$ to (1.2) which are globally Lipschitz in the space variable. Let

$$
\|u\|_{L^{\infty}\left(\mathbb{R}^{n} \times(0, T)\right)},\|D u\|_{L^{\infty}\left(\mathbb{R}^{n} \times(0, T)\right)},\|v\|_{L^{\infty}\left(\mathbb{R}^{n} \times(0, T)\right)},\|D v\|_{L^{\infty}\left(\mathbb{R}^{n} \times(0, T)\right)} \leq C .
$$

Then, there exists a constant $M=M(C)$ such that

$$
\|u-v\|_{L^{\infty}\left(\mathbb{R}^{n} \times(0, T)\right)} \leq e^{M T}\|u(\cdot, 0)-v(\cdot, 0)\|_{L^{\infty}\left(\mathbb{R}^{n} \times(0, T)\right)} .
$$

Proof. First, we let $G \in C^{2}(\mathbb{R})$ be a compactly supported real-valued function such that $G(w)=$ $F(w)$ when $|w| \leq 2 C+1$. Let now $\phi$ be a test function such that $u-\phi$ has a local extremum at a point $z_{0}=\left(x_{0}, t_{0}\right)$. From $(3.1)$, it follows that $|D \phi| \leq C$, and a similar conclusion is also true 
when $u$ is replaced by $v$. Therefore, if we define $Q(y)=|y|^{2-p}$ if $|y| \leq 2 C$ and $Q(y)=2^{2-p} C^{2-p}$ when $|y| \geq 2 C$, we have that both $u$ and $v$ are viscosity solutions to

$$
w_{t}+Q(D w) G^{\prime}(w)=\left(\delta_{i j}+(p-2) \frac{w_{i} w_{j}}{|D w|^{2}}\right) w_{i j} .
$$

This equation obeys the hypothesis of Theorem 4.1 in [GGIS]. As a consequence, (3.2) follows from a slight modification of the arguments in the proof of Theorem 4.1 in [GGIS] which can be found for instance in Theorem 1.2.1 in [Zh]. Note that the modification is similar to the one employed for the case $F=0$ in proof of Theorem 3.4 in [BG1].

\section{Existence of SOlutions}

In this section we establish the solvability of the Cauchy problem (1.2) when the initial datum $g \in C^{0,1}\left(\mathbb{R}^{n}\right)$, i.e., $g$ is globally Lipschitz and bounded. With this objective in mind, for any $\varepsilon>0$ we consider the approximating Cauchy problem

$$
\left\{\begin{array}{l}
u_{t}^{\varepsilon}+\left(\varepsilon^{2}+\left|D u^{\varepsilon}\right|^{2}\right)^{1-p / 2} f\left(u^{\varepsilon}\right)=a_{i j}^{\varepsilon}\left(D u^{\varepsilon}\right) u_{i j}^{\varepsilon} \\
u^{\varepsilon}(\cdot, 0)=g,
\end{array}\right.
$$

where we have let $f=F^{\prime}$, and

$$
a_{i j}^{\varepsilon}(\sigma)=\delta_{i j}+(p-2) \frac{\sigma_{i} \sigma_{j}}{\varepsilon^{2}+|\sigma|^{2}}, \quad i, j=1, \ldots, n .
$$

It is easily seen that for every $\sigma \in \mathbb{R}^{n}$ and every $\xi \in \mathbb{R}^{n}$ the following uniform ellipticity condition is satisfied, independently of $\varepsilon>0$,

$$
\min \{1, p-1\}|\xi|^{2} \leq a_{i j}^{\varepsilon}(\sigma) \xi_{i} \xi_{j} \leq \max \{1, p-1\}|\xi|^{2} .
$$

Proceeding as follows we first obtain a unique bounded classical solution $u^{\varepsilon}$ to (4.1).

We let $M=\|g\|_{L^{\infty}\left(\mathbb{R}^{n}\right)}$. In correspondence of the initial datum $g$ we assume that the nonlinearity $F$ in (1.2) satisfy the following hypothesis: there exist constants $q, M_{1}, M_{2}$, all depending on $M$, such that one has

$$
\left\{\begin{array}{l}
-q \leq M_{1} \leq-M \text { and } M \leq M_{2} \leq q, \\
f\left(M_{1}\right) \leq 0 \leq f\left(M_{2}\right)
\end{array}\right.
$$

We remark immediately that assumption (4.4) will be needed only in the case $p=2$, but not when $1<p<2$. We also note that for the typical representatives of nonlinearities $f(u)=u^{3}-u$, $f(u)=\sin u$ in (1.2) the assumption (4.4) is satisfied.

Assuming (4.4) let now $\tilde{F}$ be a compactly supported, $C^{2, \beta}(\mathbb{R})$ function such that $\tilde{F}=F$ for $|u| \leq 2 q+1$. We first suppose additionally that $g$ is smooth and has bounded derivatives of all orders. We take a sequence of smooth domains $\Omega^{N} \nearrow \mathbb{R}^{n}$. Given any $T>0$, we consider the finite cylinders $\Omega_{T}^{N}=\Omega^{N} \times(0, T)$, and indicate with $\partial_{p} \Omega_{T}^{N}=\left(\partial \Omega^{N} \times(0, T)\right) \cup\left(\Omega^{N} \times\{0\}\right)$ its parabolic boundary. For each $N \in \mathbb{N}$, and $\varepsilon>0$, we solve the Cauchy-Dirichlet problem

$$
\left\{\begin{array}{l}
u_{t}^{\varepsilon, N}+\left(\varepsilon^{2}+\left|D u^{\varepsilon, N}\right|^{2}\right)^{1-p / 2} \tilde{F}^{\prime}\left(u^{\varepsilon, N}\right)=a_{i j}^{\varepsilon}\left(D u^{\varepsilon, N}\right) u_{i j}^{\varepsilon, N} \text { in } \Omega_{T}^{N}, \\
\left.u^{\varepsilon, N}=g \text { on } \partial_{p} \Omega_{T}^{N} \text { (one should keep in mind that } g(x, t)=g(x)\right) .
\end{array}\right.
$$

The existence of classical solutions $u^{\varepsilon, N}$, such that $\sup _{\Omega_{T}^{N}}\left\|D u^{\varepsilon, N}\right\|<\infty$, is guaranteed by Theorem 4.2 , p. 559 in [LU]. Because of the boundedness of gradient, one can see that $u^{\varepsilon, N}$ satisfies an equation which obeys the hypothesis of the comparison principle, Theorem $9.1 \mathrm{in}$ [Li]. Moreover, because of (4.4) $M_{1}$ is a subsolution and $M_{2}$ is a supersolution to such an equation. Therefore, from the comparison principle Theorem 3.1 above we conclude that $\left|u^{\varepsilon, N}\right|$ is bounded from above by $q$, which is independent of $N$ and $\varepsilon$. Since $\tilde{F}^{\prime}(s)=f(s)$ when $|s| \leq 2 q$, we infer that 
$u^{\varepsilon, N}$ solves the Cauchy-Dirichlet problem with $\tilde{F}^{\prime}$ replaced by $f$. The rest of the proof for the existence of solutions $u^{\varepsilon}$ to the Cauchy problem corresponding to (4.1) remains the same as for the case $F=0$, see [BG1]. Since $F \in C_{l o c}^{2, \beta}(\mathbb{R})$, it follows from the Schauder theory ( see Chapter 4 and Chapter 12 in $[\mathrm{Li}])$, that $u^{\varepsilon} \in H_{3+\alpha}\left(\mathbb{R}^{n} \times[0, T]\right)$ for some $\alpha>0$ which depends on $\varepsilon, p, n, q$ and $\beta$. We refer to Chapter 4 in [Li] for relevant notion of $H_{3+\alpha}$ spaces.

We note that the solutions $u^{\varepsilon}$ 's have spatial gradient bounds, depending only on $n, p, q$ and $\|D g\|_{L^{\infty}\left(\mathbb{R}^{n}\right)}$, which are uniform in $\varepsilon$ for $\varepsilon \leq 1$. This follows from Theorem $11.3 \mathrm{~b}$ ) in [Li]. For this, one needs to observe that the limit behavior in $(11.17)$ in $[\mathrm{Li}]$ is uniform in $\varepsilon$, similarly to the case $F=0$. Now, as in the case $F=0$, the uniform bounds on the time derivatives of $u^{\varepsilon}$, which depend only on the $C^{2}$ norm of $g$, can be obtained by differentiating the approximating equations (4.1) with respect to the time variable and by applying Theorem 3.1 above. Therefore, in the same way as for the case $F=0$, one can assert the existence of $u$ to (1.2) in the class $H^{T}$ when $g$ is smooth and has bounded derivatives of all orders.

In the case when $g$ is only globally Lipschitz, we take $\varepsilon_{k}$-mollifications of $g$ for a sequence $\varepsilon_{k} \rightarrow 0$, and call them $g_{k}$. Then, $g_{k}$ has bounded derivatives of all order and

$$
\left\|g_{k}\right\|_{L^{\infty}\left(\mathbb{R}^{n}\right)} \leq\|g\|_{L^{\infty}\left(\mathbb{R}^{n}\right)}, \quad\left\|D g_{k}\right\|_{L^{\infty}\left(\mathbb{R}^{n}\right)} \leq\|D g\|_{L^{\infty}\left(\mathbb{R}^{n}\right)} .
$$

Let $u_{k}$ be the solution to the Cauchy problem corresponding to the initial datum $g_{k}$. As mentioned above, thanks to Theorem 11.3 in [Li] ensures that $\left\|D u_{k}\right\|_{L^{\infty}\left(\mathbb{R}^{n} \times(0, T)\right)}$ is bounded uniformly in $k$ by constants which depends only on $\|D g\|_{L^{\infty}\left(\mathbb{R}^{n}\right)}, q, p$ and $n$. Since $g_{k} \rightarrow g$ uniformly in $\mathbb{R}^{n}$, by the maximum modulus principle Theorem 3.1 above we conclude that $u_{k} \rightarrow u$ uniformly in $\mathbb{R}^{n} \times[0, T]$, where $u$ is the unique solution to the Cauchy problem (1.2) in the class $H^{T}$ corresponding to the initial datum $g$.

Remark 4.1. We note that the assumption (4.4) is only used to assert a bound on $u^{\varepsilon, N}$ independent of $\varepsilon$ and $N$ as an intermediate step. If we instead assume that $f$ is bounded, it turns out that $w=\|g\|_{L^{\infty}\left(\mathbb{R}^{n}\right)}+M_{1} t$ is a supersolution to the equation satisfied by $u^{\varepsilon, N}$ when $\varepsilon \leq 1$ and $M_{1}$ is chosen large enough depending only on $\|f\|_{L^{\infty}\left(\mathbb{R}^{n}\right)}$. Hence, such $w$ can be used as a barrier for $u^{\varepsilon, N}$ from above and one can similarly bound $u^{\varepsilon, N}$ from below by using $-w$ which is a subsolution to the same equation.

Remark 4.2. When $1<p<2$, the assumption (4.4) is not needed. In that case, let $\tilde{F}$ be a $C^{2, \beta}$ compactly supported function such that $\tilde{F}(s)=F(s)$ when $|s| \leq\|g\|_{L^{\infty}\left(\mathbb{R}^{n}\right)}+2$. Then, for each $\varepsilon>0$, we solve the corresponding Cauchy-Dirichlet problem as before in $\Omega_{T}^{N}$ with $\tilde{F}^{\prime}$ instead of $f$ and denote the corresponding solutions by $u^{\varepsilon, N}$. For all $\varepsilon$ small enough depending only on $p$, $f, T$ and $\|g\|_{L^{\infty}\left(\mathbb{R}^{n}\right)}$, it turns out that $w=\|g\|_{L^{\infty}\left(\mathbb{R}^{n}\right)}+\frac{t}{T}$ is a supersolution to the equation satisfied by $u^{\varepsilon, N}$ and hence can be used to assert boundedness of $u^{\varepsilon, N}$ from above. Similarly, the subsolution $-w$ can be used to assert boundedness for $u^{\varepsilon, N}$ from below. Therefore, for all such small enough $\varepsilon$, it follows from the definition of $\tilde{F}$ that $u^{\varepsilon, N}$ solves the Cauchy-Dirichlet problem with $\tilde{F}^{\prime}$ replaced with $f$. The rest of the proof remains the same. This procedure does not work in the case $p=2$. This is because when the approximating equation (4.5) is computed for $w$, the term $\varepsilon^{2-p} \tilde{F}^{\prime}(w)$ does not go to zero as $\varepsilon \rightarrow 0$ in the case $p=2$ and therefore one cannot assert that $w$ is a supersolution to (4.5). Therefore, one interesting aspect is that for $1<p<2$, one has existence of solution to the Cauchy problem (1.2) without any growth assumption on $f$ due to the special structure of the equation unlike what one needs in the general theory of uniformly parabolic equations, see for instance Theorem 12.16 in [Li].

\section{Proof of the Main Results}

We first prove an intermediate crucial result which asserts gradient estimates for solutions to the approximating Cauchy problems (4.1). For each $\varepsilon>0$, we define

$$
P_{\varepsilon}\left(u^{\varepsilon}\right)(x, s)=\xi_{\varepsilon}\left(\left|D u^{\varepsilon}(x, s)\right|^{2}\right)-2 F\left(u^{\varepsilon}(x, s)\right),
$$


where $u^{\varepsilon}$ is a solution to (4.1), and we have let

$$
\xi_{\varepsilon}(s)=2 s \phi_{\varepsilon}^{\prime}-\phi_{\varepsilon}, \quad \text { with } \quad \phi_{\varepsilon}(s)=\frac{2}{p}\left(\varepsilon^{2}+s\right)^{p / 2} .
$$

Theorem 5.1. Let $u^{\varepsilon}$ be a solution of the approximating equation (4.1) such that $u^{\varepsilon} \in H_{3+\alpha}\left(\mathbb{R}^{n} \times\right.$ $[0, T])$ for some $\alpha>0$. If $P_{\varepsilon}\left(u^{\varepsilon}\right)(\cdot, 0) \leq 0$, then $P_{\varepsilon}\left(u^{\varepsilon}(x, t)\right) \leq 0$ for all $x \in \mathbb{R}^{n}$ and all $t \geq 0$.

Remark 5.2. Note that, when the initial datum $g$ has bounded derivatives of sufficiently high order (up to order five), then the solutions $u^{\varepsilon}$ constructed in Section 4 satisfy the hypothesis of Theorem 5.1.

Proof of Theorem 5.1. Henceforth, we will routinely omit $\varepsilon$-subscripts and superscripts, and suppress the dependence of $P$ on $u$. Thus, for instance, we will write $u$ instead of $u^{\varepsilon}, P$ instead of $P_{\varepsilon}\left(u^{\varepsilon}\right)$. We will also write $\phi$ and $\xi$, instead of $\phi_{\varepsilon}$ and $\xi_{\varepsilon}$ like in (5.2). Note that the approximating equation can be rewritten as

$$
\operatorname{div}\left(\phi^{\prime}\left(|D u|^{2}\right) D u\right)=f(u)+\phi^{\prime}\left(|D u|^{2}\right) u_{t} .
$$

We let $\Lambda=\xi^{\prime}$, and note that for each $\varepsilon>0$ we have from (5.2)

$$
\Lambda=\left(\varepsilon^{2}+|D u|^{2}\right)^{p / 2-2}\left(\varepsilon^{2}+(p-1)|D u|^{2}\right)>0 .
$$

We next write (5.3) in the following manner

$$
a_{i j}(D u) u_{i j}=f(u)+\phi^{\prime} u_{t}
$$

where

$$
a_{i j}=2 \phi^{\prime \prime} u_{i} u_{j}+\phi^{\prime} \delta_{i j}
$$

Therefore, $u$ satisfies

$$
d_{i j} u_{i j}=\frac{f}{\Lambda}+\frac{\phi^{\prime}}{\Lambda} u_{t}
$$

where $d_{i j}=\frac{a_{i j}}{\Lambda}$. By differentiating (5.5) with respect to $x_{k}$, we obtain

$$
\left(a_{i j}\left(u_{k}\right)_{i}\right)_{j}=f^{\prime} u_{k}+\phi^{\prime} u_{t k}+2 \phi^{\prime \prime} u_{h k} u_{h} u_{t} .
$$

From the definition of $P$ in (5.1) we have,

$$
P_{i}=2 \Lambda u_{k i} u_{k}-2 f u_{i}, \quad P_{t}=2 \Lambda u_{k t} u_{k}-2 f u_{t} .
$$

We now consider the following auxiliary function

$$
w=w_{R}=P-\frac{M}{R} \sqrt{|x|^{2}+1}-\frac{c t}{R^{1 / 2}},
$$

where $R>1$ and $M, c$ are to be determined subsequently. Note that $P \geq w$ for $t \geq 0$. Consider the cylinder $Q_{R}=B(0, R) \times[0, T]$. One can see that if $M$ is chosen large enough, depending on the $L^{\infty}$ norm of $u$ and its first derivatives, then $w<0$ on the lateral boundary of $Q_{R}$. In this situation we see that if $w$ has a strictly positive maximum at a point $\left(x_{0}, t_{0}\right)$, then such point cannot be on the parabolic boundary of $Q_{R}$. In fact, since $w<0$ on the lateral boundary, the point cannot be on such set. But it cannot be on the bottom of the cylinder either since at $t=0$ we have $w(\cdot, 0) \leq P(u(\cdot, 0))=P(g) \leq 0$, where in the last inequality we have used the hypothesis.

Our objective is to prove the following claim:

$$
w \leq K \stackrel{\text { def }}{=} R^{-\frac{p}{2}}, \quad \text { in } Q_{R},
$$

provided that $M$ and $c$ are chosen appropriately. This claim will be established in (5.30) below. We first fix a point $(y, s)$ in $\mathbb{R}^{n}$. Now for all $R$ sufficiently large enough, we have that $(y, s) \in Q_{R}$. 
We would like to emphasize over here that finally we let $R \rightarrow \infty$. Therefore, once (5.9) is established, we obtain from it and the definition of $w$ that

$$
P(u)(y, s) \leq \frac{K^{\prime}}{R^{1 / 2}}
$$

where $K^{\prime}$ depends on $\varepsilon,(y, s)$ and the bounds of the derivatives of $u$ of order three. By letting $R \rightarrow \infty$ in (5.10), we find that

$$
P(u)(y, s) \leq 0 .
$$

The sought for conclusion thus follows from the arbitrariness of the point $(y, s)$.

In order to prove the claim (5.9) we argue by contradiction and suppose that there exist $\left(x_{0}, t_{0}\right) \in \bar{Q}_{R}$ at which $w$ attains it maximum and for which

$$
w\left(x_{0}, t_{0}\right)>K \text {. }
$$

This implies that $\left(x_{0}, t_{0}\right)$ is not on the parabolic boundary of $Q_{R}$. Note that from the definition (5.1) of $P$, we have

$$
\frac{1}{2} P=\left(\varepsilon^{2}+|D u|^{2}\right)^{\frac{p}{2}-1}\left[\frac{1}{p^{\prime}}|D u|^{2}-\frac{\varepsilon^{2}}{p}\right]-F(u) .
$$

Since $1<p \leq 2$, we have $2 \leq p^{\prime}<\infty$, and so $\frac{1}{p^{\prime}} \leq \frac{1}{2}<1$. Thus, at every point of $Q_{R}$ we have

$$
\frac{1}{2} w \leq \frac{1}{2} P \leq \frac{1}{p^{\prime}}\left(\varepsilon^{2}+|D u|^{2}\right)^{\frac{p}{2}-1}|D u|^{2}<\left(\varepsilon^{2}+|D u|^{2}\right)^{\frac{p}{2}-1}|D u|^{2} .
$$

It follows that at $\left(x_{0}, t_{0}\right)$ we must have

$$
\left(\varepsilon^{2}+\left|D u\left(x_{0}, t_{0}\right)\right|^{2}\right)^{\frac{p}{2}-1}\left|D u\left(x_{0}, t_{0}\right)\right|^{2} \geq \frac{1}{2} P\left(x_{0}, t_{0}\right) \geq \frac{1}{2} w\left(x_{0}, t_{0}\right)>\frac{1}{2} K,
$$

which implies, in particular, that $D u\left(x_{0}, t_{0}\right) \neq 0$. Therefore, since $1<p \leq 2$, we obtain from

$$
\left|D u\left(x_{0}, t_{0}\right)\right|^{p} \geq\left(\varepsilon^{2}+\left|D u\left(x_{0}, t_{0}\right)\right|^{2}\right)^{p / 2-1}\left|D u\left(x_{0}, t_{0}\right)\right|^{2} \geq \frac{1}{2} P\left(x_{0}, t_{0}\right)>\frac{1}{2} K .
$$

On the other hand, since $\left(x_{0}, t_{0}\right)$ does not belong to the parabolic boundary, from the hypothesis that $w$ has its maximum at such point, we conclude that $w_{t}\left(x_{0}, t_{0}\right) \geq 0$ and $D w\left(x_{0}, t_{0}\right)=0$. These conditions translate into

$$
P_{t} \geq \frac{c}{R^{1 / 2}}
$$

and

$$
P_{i}=\frac{M}{R} \frac{x_{0, i}}{\left(\left|x_{0}\right|^{2}+1\right)^{1 / 2}}
$$

Now

where

$$
\left(d_{i j} P_{i}\right)_{j}=2\left(\frac{a_{i j}}{\Lambda}\left(\Lambda u_{k i} u_{k}-f u_{i}\right)\right)_{j}=2\left(a_{i j}\left(u_{k}\right)_{i} u_{k}\right)_{j}-2\left(f d_{i j} u_{i}\right)_{j} .
$$

After a simplification, (5.16) equals

$$
2 a_{i j}\left(u_{k i}\right)_{j} u_{k}+2 a_{i j} u_{k i} u_{k j}-2 f^{\prime} d_{i j} u_{i} u_{j}-2 f d_{i j} u_{i j}-2 f\left(d_{i j}\right)_{j} u_{i} .
$$

We notice that

$$
d_{i j} u_{i} u_{j}=\frac{2 \phi^{\prime \prime} u_{i} u_{j} u_{i} u_{j}+\phi^{\prime} \delta_{i j} u_{i} u_{j}}{\Lambda}=|D u|^{2} .
$$


Now by using (5.7) and by cancelling the term $2 f^{\prime}|D u|^{2}$, we get that the right-hand side in (5.16) equals

$$
2 \phi^{\prime} u_{t k} u_{k}+4 \phi^{\prime \prime} u_{h k} u_{h} u_{k} u_{t}+2 a_{i j} u_{k i} u_{k j}-2 f d_{i j} u_{i j}-2 f d_{i j, j} u_{i} .
$$

Therefore by using the equation (5.6), we obtain

$$
\begin{aligned}
\left(d_{i j} P_{i}\right)_{j} & =2 a_{i j} u_{k i} u_{k j}+2 \phi^{\prime} u_{t k} u_{k}+4 \phi^{\prime \prime} u_{h k} u_{h} u_{k} u_{t} \\
& -2 \frac{f^{2}}{\Lambda}-2 \frac{f \phi^{\prime} u_{t}}{\Lambda}-2 f d_{i j, j} u_{i} .
\end{aligned}
$$

By using the extrema conditions (5.14), (5.15), we have the following two conditions at $\left(x_{0}, t_{0}\right)$

$$
\begin{gathered}
u_{k h} u_{k} u_{h}=\frac{f}{\Lambda}|D u|^{2}+\frac{M}{2 R \Lambda} \frac{x_{h} u_{h}}{\left(|x|^{2}+1\right)^{1 / 2}}, \\
2 \Lambda u_{k t} u_{k} \geq 2 f u_{t}+\frac{c}{R^{1 / 2}} .
\end{gathered}
$$

Using the extrema conditions and by canceling $2 \phi^{\prime} u_{t k} u_{k}$ we obtain,

$$
\begin{aligned}
\left(d_{i j} w_{i}\right)_{j} & \geq 2 a_{i j} u_{k i} u_{k j}+\frac{4 \phi^{\prime \prime} f}{\Lambda}|D u|^{2} u_{t}-\frac{2 f^{2}}{\Lambda}-2 f d_{i j, j} u_{i} \\
& +\frac{2 \phi^{\prime \prime} M x_{h} u_{h} u_{t}}{R \Lambda\left(|x|^{2}+1\right)^{1 / 2}}+\frac{c \phi^{\prime}}{R^{1 / 2} \Lambda}-\frac{M}{R}\left(d_{i j} \frac{x_{i}}{\left(|x|^{2}+1\right)^{1 / 2}}\right)_{j} .
\end{aligned}
$$

Now we have the following structure equation, whose proof is lengthy but straightforward,

$$
d_{i j, j} u_{i}=\frac{2 \phi^{\prime \prime}}{\Lambda}\left(|D u|^{2} \Delta u-u_{h k} u_{h} u_{k}\right) .
$$

Using (5.19) in (5.21), we find

$$
d_{i j, i} u_{i}=\frac{2 \phi^{\prime \prime}|D u|^{2}}{\Lambda}\left(\Delta u-\frac{f}{\Lambda}-\frac{M x_{h} u_{h}}{2 R|D u|^{2} \Lambda\left(|x|^{2}+1\right)^{1 / 2}}\right) .
$$

Using the equation (5.3), we have

$$
2 \phi^{\prime \prime} u_{h k} u_{h} u_{k}+\phi^{\prime} \Delta u=f+\phi^{\prime} u_{t} .
$$

Therefore,

$$
\Delta u=\frac{f+\phi^{\prime} u_{t}-2 \phi^{\prime \prime} u_{h k} u_{h} u_{k}}{\phi^{\prime}} .
$$

Substituting the value for $\Delta u$ in (5.22) and by using the extrema condition (5.19), we have the following equality at $\left(x_{0}, t_{0}\right)$,

$$
\begin{aligned}
d_{i j, j} u_{i} & =\frac{2 \phi^{\prime \prime}|D u|^{2}}{\Lambda \phi^{\prime}}\left[f+u_{t} \phi^{\prime}-2 \phi^{\prime \prime} \frac{|D u|^{2}}{\Lambda} f-f \frac{\phi^{\prime}}{\Lambda}\right. \\
& \left.-\frac{\phi^{\prime \prime} M x_{h} u_{h}}{R \Lambda\left(|x|^{2}+1\right)^{1 / 2}}-\frac{M x_{h} u_{h} \phi^{\prime}}{2 R|D u|^{2} \Lambda\left(|x|^{2}+1\right)^{1 / 2}}\right] .
\end{aligned}
$$

Using the definition of $\Lambda$ and cancelling terms in (5.23), we have that the right-hand side in (5.23) equals

$$
2 \phi^{\prime \prime} \frac{|D u|^{2} u_{t}}{\Lambda}-\frac{\phi^{\prime \prime} M x_{h} u_{h}}{\Lambda^{2} R\left(|x|^{2}+1\right)^{1 / 2}}-\frac{2\left(\phi^{\prime \prime}\right)^{2}|D u|^{2} M x_{h} u_{h}}{R \Lambda^{2} \phi^{\prime}\left(|x|^{2}+1\right)^{1 / 2}} .
$$


Therefore, by canceling the terms $4 \phi^{\prime \prime} f \frac{|D u|^{2} u_{t}}{\Lambda}$ in (5.20), we obtain the following differential inequality at $\left(x_{0}, t_{0}\right)$,

$$
\begin{aligned}
\left(d_{i j} w_{i}\right)_{j} \geq & \frac{c \phi^{\prime}}{R^{1 / 2} \Lambda}-\frac{2 f^{2}}{\Lambda}-\frac{M}{R}\left(d_{i j} \frac{x_{i}}{\left(|x|^{2}+1\right)^{1 / 2}}\right)_{j}+\frac{2 \phi^{\prime \prime} M x_{h} u_{h} u_{t}}{R \Lambda\left(|x|^{2}+1\right)^{1 / 2}} \\
& +\frac{2 f \phi^{\prime \prime} M x_{h} u_{h}}{\Lambda^{2} R\left(|x|^{2}+1\right)^{1 / 2}}+\frac{4 f\left(\phi^{\prime \prime}\right)^{2}|D u|^{2} M x_{h} u_{h}}{R \Lambda^{2} \phi^{\prime}\left(|x|^{2}+1\right)^{1 / 2}}+2 a_{i j} u_{k i} u_{k j} .
\end{aligned}
$$

Now by using the identity for $D P$ in (5.8) above, we have

$$
u_{k i} u_{k j} u_{i} u_{j}=\frac{\left(P_{k}+2 f u_{k}\right)^{2}}{4 \Lambda^{2}} .
$$

Also,

$$
a_{i j} u_{k j} u_{k i}=\phi^{\prime} u_{i k} u_{i k}+2 \phi^{\prime \prime} u_{i k} u_{i} u_{j k} u_{j}
$$

Therefore, by Schwarz inequality, we have

$$
a_{i j} u_{k j} u_{k i} \geq \phi^{\prime} \frac{u_{i k} u_{j k} u_{i} u_{j}}{|D u|^{2}}+2 \phi^{\prime \prime} u_{i k} u_{i} u_{j k} u_{j}=\frac{\Lambda u_{i k} u_{i} u_{j k} u_{j}}{|D u|^{2}} .
$$

Then, by using (5.26) we find

$$
a_{i j} u_{k j} u_{k i} \geq \frac{\left(P_{k}+2 f u_{k}\right)^{2}}{4 \Lambda|D u|^{2}}=\frac{|D P|^{2}+4 f^{2}|D u|^{2}+2 f<D u, D P>}{4|D u|^{2} \Lambda} .
$$

At this point, using (5.27) in (5.25), we can cancel off $\frac{2 f^{2}}{\Lambda}$ and consequently obtain the following inequality at $\left(x_{0}, t_{0}\right)$,

$$
\begin{aligned}
\left(d_{i j} w_{i}\right)_{j} \geq & \frac{c \phi^{\prime}}{R^{1 / 2} \Lambda}+\frac{f<D u, D P>}{|D u|^{2} \Lambda}-\frac{M}{R}\left(d_{i j} \frac{x_{i}}{\left(|x|^{2}+1\right)^{1 / 2}}\right)_{j}+\frac{2 \phi^{\prime \prime} M x_{h} u_{h} u_{t}}{R \Lambda\left(|x|^{2}+1\right)^{1 / 2}} \\
& +\frac{4 f\left(\phi^{\prime \prime}\right)^{2}|D u|^{2} M x_{h} u_{h}}{R \Lambda^{2} \phi^{\prime}\left(|x|^{2}+1\right)^{1 / 2}}+\frac{2 f \phi^{\prime \prime} M x_{h} u_{h}}{\Lambda^{2} R\left(|x|^{2}+1\right)^{1 / 2}} .
\end{aligned}
$$

By assumption, since $w\left(x_{0}, t_{0}\right) \geq K$, we have that

$$
|D u| \geq \frac{1}{2^{1 / p} R^{1 / 2}} .
$$

Moreover, since $u$ has bounded derivatives of upto order 3 , for a fixed $\varepsilon>0$, we have that $\phi^{\prime}$ and $\Lambda$ are bounded from below by a positive constant. Therefore by (5.15), the term $\frac{f\langle D u, D P\rangle}{|D u|^{2} \Lambda}$ can be controlled from below by $-\frac{M^{\prime \prime}}{R^{1 / 2}}$ where $M^{\prime \prime}$ depends on $\varepsilon$ and the bounds of the derivatives of $u$. Consequently, from (5.28), we have at $\left(x_{0}, t_{0}\right)$,

$$
\left(d_{i j} w_{i}\right)_{j} \geq \frac{C(c)}{R^{1 / 2}}-\frac{L(M)}{R}-\frac{M^{\prime \prime}}{R^{1 / 2}} .
$$

Now in the very first place, if $c$ is chosen large enough depending only on $\varepsilon$ and the bounds of the derivatives of $u$ up to order three, we would have the following inequality at $\left(x_{0}, t_{0}\right)$,

$$
\left(d_{i j} w_{i}\right)_{j}>0 .
$$

This contradicts the fact that $w$ has a maximum at $\left(x_{0}, t_{0}\right)$. Therefore, either $w\left(x_{0}, t_{0}\right)<K$, or the maximum of $w$ is achieved on the parabolic boundary where $w<0$. In either case, for an arbitrary point $(y, s)$ such that $|y| \leq R$, we have that

$$
w(y, s) \leq \frac{1}{R^{p / 2}} .
$$


Proof of Theorem 1.1. Let $g_{k}$ be the $\varepsilon_{k}$ mollifications of $g$ which converges to $g$ uniformly in $\mathbb{R}^{n}$ as $k \rightarrow \infty$. Note that $g_{k}$ has bounded derivatives of all orders with bounds depending on $\varepsilon_{k}$. Given any $\delta>0$, we note that for large enough $k, g_{k}$ satisfies (1.6) with $F$ replaced by $G=F+\delta$. This can be seen as follows:

$$
\left|D g_{k}(x)\right|=\left|\int_{\mathbb{R}^{n}} D g(x-y) \rho_{\varepsilon_{k}}(y) d y\right| \leq \int_{\mathbb{R}^{n}}|D g(x-y)| \rho_{\varepsilon_{k}}(y) d y
$$

See for instance Theorem 6.25 in $[R]$. We choose to cite this reference since the integrals considered in (5.31) are vector valued and we need to make sure that no additional constants are incurred in front of the last integral in (5.31). Therefore,

$$
\left|D g_{k}(x)\right|^{p}=\left|\int_{\mathbb{R}^{n}} D g(x-y) \rho_{\varepsilon_{k}}(y) d y\right|^{p} \leq \int_{\mathbb{R}^{n}}|D g(x-y)|^{p} \rho_{\varepsilon_{k}}(y) d y
$$

The last inequality in (5.32) follows from (5.31) and Jensen inequality. Now since $|D g|^{p} \leq$ $\frac{p}{p-1} F(g)$ a.e., we have for all $k$ large enough,

$$
\left|D g_{k}(x)\right|^{p} \leq \frac{p}{p-1} \int_{\mathbb{R}^{n}} F(g(x-y)) \rho_{\varepsilon_{k}}(y) d y \leq \frac{p}{p-1} \sup _{B_{\varepsilon_{k}}(x)} F(g) \leq \frac{p}{p-1}\left(F\left(g_{k}(x)\right)+\delta\right) .
$$

In the last inequality in (5.33), we have made use of the fact that $g_{k}$ converges to $g$ uniformly in $\mathbb{R}^{n}$ since $g$ is globally Lipschitz. This justifies the claim above.

Now for each such $k$, let $u_{k}^{\varepsilon}$ be the solution to the Cauchy problem corresponding to equation (4.1) with initial datum $g_{k}$. We furthermore assume that for $1<p<2, \varepsilon$ is small enough so that the conditions in Remark 4.2 is satisfied. We now note that for $1<p \leq 2$,

$$
\tilde{P}_{\varepsilon} \leq \tilde{P}
$$

where $\tilde{P}, \tilde{P}_{\varepsilon}$ are defined as in (5.1). Therefore, since $\tilde{P}\left(g_{k}\right) \leq 0$, we have that $\tilde{P}_{\varepsilon}\left(g_{k}\right) \leq 0$. Theorem 5.1 applied to $u_{k}^{\varepsilon}$ implies that $\tilde{P}_{\varepsilon}\left(u_{k}^{\varepsilon}\right) \leq 0$ for all positive times. Now, by Dini's theorem the functions $h_{\varepsilon}(x)=\left(\varepsilon^{2}+|x|^{2}\right)^{p / 2-1}|x|^{2} \rightarrow|x|^{p}$, uniformly on compact sets. Thus, because of uniform bounds on the gradients, given any $\gamma>0$ for all small enough $\varepsilon$ we have that at each time level $t$,

$$
\frac{p-1}{p}\left|D u_{k}^{\varepsilon}\right|^{p} \leq G\left(u_{k}^{\varepsilon}\right)+\gamma .
$$

Integrating (5.35) over an open ball $B_{r}=B_{r}(x)$ where $x$ is any arbitrary point, by using lower semicontinuity on the left-hand side, and by passing to the limit in $\varepsilon$ on the right-hand side, and then by letting $\gamma \rightarrow 0$, we find

$$
\frac{p-1}{p} \int_{B_{r}}\left|D u_{k}\right|^{p} \leq \int_{B_{r}} G\left(u_{k}\right)
$$

Now by the maximum modulus principle, Theorem $3.1, u_{k} \rightarrow u$ uniformly in $\mathbb{R}^{n}$ and weakly in $W_{l o c}^{1, p}\left(\mathbb{R}^{n}\right)$ at any given time $t$ where $u$ is the solution to the Cauchy problem with initial datum $g$. Therefore in (5.36), by using lower semicontinuity on the left hand side and by passing to the limit in $k$ on the right hand side, we have that (5.36) holds for $u$. Then from the Lebesgue differentiation theorem, it follows that at a given time level $t$,

$$
\frac{p-1}{p}|D u|^{p} \leq F(u)+\delta \quad \text { a.e. in } \mathbb{R}^{n} .
$$

By letting $\delta \rightarrow 0$, we reach the desired conclusion.

Proof of Theorem 1.5. Since by hypothesis, $g$ has bounded derivatives of up to order 2, we have by an application of maximum principle as described in Section 4 , that the solutions $u^{\varepsilon}$ to the approximating equations (4.1) are such that $\left|D u^{\varepsilon}\right|$ and $\left|u_{t}^{\varepsilon}\right|$ are bounded from above by constants 
which are independent of $\varepsilon$. Therefore, in the case $n=2$, we see that, because of uniform bounds on the space and time derivatives, at each time level $t$ the solutions $u^{\varepsilon}$ to the approximating equations (4.1) solve a uniformly elliptic linear PDE in non-divergence form with right-hand side uniformly bounded in $\varepsilon$. Therefore, from Theorem 12.4 in [GT] (see also [T]), it follows that $D u^{\varepsilon}(\cdot, t)$ has uniform Hölder bounds independent of $\varepsilon$ with an exponent $\alpha$ which only depends on $p$ (note that Theorem 12.4 is very specific to $n=2$ because in higher dimensions the Krylov-Safonov estimates only ensure uniform $C^{\alpha}$, but not $C^{1, \alpha}$ bounds). Consequently, $D u(\cdot, t)$ is Hölder continuous in $x$ and the conclusion follows.

Now we turn our attention to the proof of Theorem 1.6 which is similar to that for the elliptic case in [CGS].

Proof of Theorem 1.6. Via an approximation argument as used before in the proof of Theorem 1.1, we can assume that the the initial datum $g$ has bounded derivatives of sufficiently high order. Let $u^{\varepsilon}$ be the solution to (4.1) corresponding to initial datum $g$. We consider the function

$$
\psi_{\varepsilon}(s)=u^{\varepsilon}\left(x_{1}+s \omega, t_{0}\right)-u^{\varepsilon}\left(x_{1}, t_{0}\right)
$$

for some $x_{1} \in \mathbb{R}^{n}$ where $\omega$ is some unit direction. The point $x_{1}$ is going to be chosen appropriately later. From the definition, we have that $\psi_{\varepsilon}(0)=0$ and

$$
\left|\psi_{\varepsilon}^{\prime}(s)\right| \leq\left|D u^{\varepsilon}\left(x_{1}+s \omega, t_{0}\right)\right| .
$$

We now define the function

For $\delta$ small enough, let

$$
\xi^{\varepsilon}(s)=2 s \phi_{\varepsilon}^{\prime}-\phi_{\varepsilon}+\frac{2}{p} \varepsilon^{2} .
$$

$$
G_{\varepsilon}=\xi^{\varepsilon}-\delta\left(\varepsilon^{2}+s\right)^{p / 2} .
$$

Clearly, $G_{\varepsilon}(0)=-\delta \varepsilon^{p}$ and by the ellipticity it is easily seen that $G_{\varepsilon}^{\prime} \geq 0$. This implies that

$$
G_{\varepsilon}(s) \geq-\delta \varepsilon^{p}
$$

Therefore from (5.38) and the definition of $G_{\varepsilon}$, given any $\gamma>0$, for small enough $\varepsilon$,

$$
\left|D u^{\varepsilon}\left(x_{1}+s \omega, t_{0}\right)\right|^{p} \leq C \xi^{\varepsilon}\left(\left|D u^{\varepsilon}\left(x_{1}+s \omega, t_{0}\right)\right|^{2}\right)+\delta \varepsilon^{p}+\gamma .
$$

By applying Theorem 5.1, we thus obtain

$$
\left|\psi_{\varepsilon}^{\prime}(s)\right|^{p} \leq C\left(F\left(u^{\varepsilon}\left(x_{1}+s \omega, t_{0}\right)\right)+k(\varepsilon)+\gamma,\right.
$$

where $k(\varepsilon) \rightarrow 0$ as $\varepsilon \rightarrow 0$. Repeating the arguments in the proof of Theorem 1.1, and finally letting $\gamma \rightarrow 0$, we obtain

$$
\left|\psi^{\prime}(s)\right|^{p} \leq C F\left(u\left(x_{1}+s \omega, t_{0}\right)\right) \quad \text { a.e. in } \mathrm{s}
$$

where

$$
\left.\psi(s)=u\left(x_{1}+s \omega, t_{0}\right)\right)-u\left(x_{1}, t_{0}\right) .
$$

Now suppose that $F\left(u\left(x_{0}, t_{0}\right)\right)=0$, and let $u_{0}=u\left(x_{0}, t_{0}\right)$. Indicating with $\Pi_{x}$ the projection onto the $x$-component, consider the set $V=\Pi_{x}\left(u^{-1}\left(u_{0}\right) \cap \mathbb{R}^{n} \times\left\{t_{0}\right\}\right)$, and let $x_{1} \in V$. Clearly, $V$ is closed. Since $F \geq 0$ and $\left.F\left(u_{0}\right)\right)=F\left(u\left(x_{1}, t_{0}\right)\right)=0$, we have that

$$
F\left(u-u_{0}\right)=O\left(\left(u-u_{0}\right)^{2}\right)
$$

Hence for $s$ small enough,

$$
F\left(u\left(x_{1}+s \omega, t_{0}\right)\right) \leq K|\psi(s)|^{2} .
$$

Therefore from (5.40), we have for all such $s$ in a small enough interval which does not depend on $\omega$,

$$
\left|\psi^{\prime}(s)\right| \leq C|\psi(s)| \text { a.e. }
$$


This implies $\psi=0$ in that same interval. Since $\omega$ is arbitrary, this implies that $V$ is open and hence equals the whole of $\mathbb{R}^{n}$. The desired conclusion thus follows.

Remark 5.3. We would like the reader to note that the reason for which we employ the regularization scheme $u^{\varepsilon}$ 's which are solutions to (4.1) in the proof of Theorem 1.6 as an intermediate step is because we can only assert that the corresponding gradient estimate (1.7) for u holds a.e. in $\mathbb{R}^{n}$. Therefore, it does not follow from Theorem 1.1 that the gradient estimate holds on the 1- dimensional line $\{x+s \omega \mid s \in \mathbb{R}\}$, which is of Lebesgue measure zero.

\section{REFERENCES}

[AC] L. Ambrosio \& X. Cabré, Entire solutions of semilinear elliptic equations in $\mathbb{R}^{3}$ and a conjecture of De Giorgi, J. Amer. Math. Soc. 13 (2000), no. 4, 725-739.

[BG1] A. Banerjee \& N. Garofalo, Gradient bounds and monotonicity of the energy for some nonlinear singular diffusion equations, Indiana Univ. Math. J. 62 (2013), no. 2, 699-736.

[BG2] A. Banerjee \& N. Garofalo, On the Dirchlet boundary value problem for the normalized p-Laplacian evolution,Commun. Pure Appl. Anal. 14 (2015), no. 1-21.

[CGS] L. Caffarelli, N. Garofalo, F. Segala, A gradient bound for entire solutions of quasilinear equations and its consequences, Comm. Pure Appl. Math. 47 (1994), 1457-1473.

[CGSpc] L. Caffarelli, N. Garofalo, F. Segala, private communication.

[CGG] Y.-G. Chen, Y. Giga \& S. Goto, Uniqueness and existence of viscosity solutions of generalized mean curvature flow equations, J. Differential Geom. 33 (1991), 749-786.

[CNV] A. Cessaroni, M. Novaga \& E. Valdinoci, A symmetry result for the Ornstein-Uhlenbeck operator., Discrete Contin. Dyn. Syst. 34 (2014), no. 6, 2451- 2467.

[DG] D. Danielli \& N. Garofalo, Properties of entire solutions of non-uniformly elliptic equations arising in geometry and in phase transitions, Calc. Var. Partial Differential Equations 15 (2002), no. 4, 451-491.

[dPKW] M. del Pino, M. Kowalczyk \& J. Wei, On De Giorgi's conjecture in dimension $N \geq 9$, Ann. of Math. (2) 174 (2011), no. 3, 1485-1569.

[dG] Giorgi, E de, Convergence problems for functionals and operators, pp 131- 188 in: Proc. Int. Meeting on Recent Methods in Nonlinear Analysis, Rome 1978, E. de Giorgi, E. Magenes, and U. Mosco, eds., Pitagora, Bologna, 1979.

[Do] K. Does, An evolution equation involving the normalized p-Laplacian, Comm. Pure Appl. Anal. 10 (2011), no. $1,361-396$.

[FSV] A. Farina, B. Sciunzi \& E. Valdinoci, Bernstein and De Giorgi type problems: new results via a geometric approach. (English summary), Ann. Sc. Norm. Super. Pisa Cl. Sci. (5) 7 (2008), no. 4, 741-791 58E12 (35B05 35J60 35J70 49J45)

[FV] A. Farina \& E. Valdinoci, A pointwise gradient estimate in possibly unbounded domains with nonnegative mean curvature., Adv. Math. 225 (2010), no. 5, 2808- 2827.

[FV1] A. Farina \& E. Valdinoci, A pointwise gradient bound for elliptic equations on compact manifolds with nonnegative Ricci curvature, Discrete Contin. Dyn. Syst. 30 (2011), no. 4, 1139-1144.

[FV2] A. Farina \&. E. Valdinoci, The state of the art for a conjecture of De Giorgi and related problems, Recent progress on reaction-diffusion systems and viscosity solutions, 74-96, World Sci. Publ., Hackensack, NJ, 2009. 35J60 (35B05)

[GG1] N. Ghoussoub \& C. Gui, On a conjecture of De Giorgi and some related problems, Math. Ann. 311 (1998), no. 3, 481-491.

[GG2] N. Ghoussoub \& C. Gui, On De Giorgi's conjecture in dimensions 4 and 5, Ann. of Math. (2) 157 (2003), no. $1,313-334$.

[GGIS] Y. Giga, S. Goto, H. Ishii \& M. Sato, Comparison principle and convexity preserving properties for singular degenerate parabolic equations on unbounded domains, Indiana Univ. Math. J. 40 (1991), 443-470.

[GT] D. Gilbarg \& N. Trudinger, Elliptic Partial Differential Equations of Second Order, Second edition. Grundlehren der Mathematischen Wissenschaften [Fundamental Principles of Mathematical Sciences], 224. Springer-Verlag, Berlin, 1983. xiii+513 pp.

[Ju] P. Juutinen, Decay estimates in sup norm for the solutions to a nonlinear evolution equation, Proceedings of the Royal Society of Edinburgh: Section A Mathematics 144 (2014), no. 3, 557-566.

[JK] P. Juutinen \& B. Kawohl, On the evolution governed by the infinity Laplacian, Math. Ann. 335 (2006), no. $4,819-851$.

[Li] G. Lieberman, Second order parabolic differential equations, World Scientific Publishing Co., Inc., River Edge, NJ, 1996. xii+439 pp. 
[LU] O. Ladyzhenskaja, V. A. Solonnikov \& N. Uraltseva, Linear and quasilinear equations of parabolic type, Translations of Mathematical Monographs, Vol. 23 American Mathematical Society, Providence, R.I., 1967.

[MPR] J. J. Manfredi, M. Parviainen \& J. D. Rossi,An asymptotic mean value characterization for a class of nonlinear parabolic equations related to tug of war games, SIAM J. Math Anal. 42 (2010), 2058-2081.

[Mo] L. Modica, A gradient bound and a Liouville theorem for nonlinear Poisson equations, Comm. Pure Appl. Math. 38 (1985), 679-684.

[R] W. Rudin, Principles of mathematical analysis Third edition. International Series in Pure and Applied Mathematics. McGraw-Hill Book Co., New York-Auckland-Dsseldorf, 1976.

[Sa] O. Savin, Regularity of flat level sets in phase transitions, Ann. of Math. (2) 169 (2009), no. 1, 41-78.

[SSV] O. Savin, B. Sciunzi \& E. Valdinoci, Flat level set regularity of p-Laplace phase transitions., Mem. Amer. Math. Soc. 182 (2006), no. 858, vi+144 pp.

[T] G. Talenti, Equazioni lineari ellittiche in due variabili. Matematiche (Catania) 21 (1966), 339-376.

[W] Lihe Wang, On the regularity theory of fully nonlinear parabolic equations: I, Comm. Pure Appl. Math. 45 (1992), 27-76.

[Zh] Y. Zhan, Viscosity Solutions of Nonlinear Degenerate Parabolic Equations and Several Applications, Ph. D. thesis, University of Toronto (1999).

Department of Mathematics, University of California, Irvine, CA- 92697

E-mail address, Agnid Banerjee: agnidban@gmail.com

Dipartimento di Ingegneria Civile, Edile e Ambientale (DiCEA), Università di Padova, 35131 PADOVA, ITALY

E-mail address, Nicola Garofalo: rembdrandt54@gmail.com 